\title{
Mobile RSVP with Dynamic Resource Sharing
}

\author{
Ali Mahmoodian ${ }^{1}$, Günter Haring ${ }^{2}$ \\ Institute for Applied Computer Science and Information Systems, Department for Advanced \\ Computer Engineering, Vienna University \\ Lenaugasse 2/8, A-1080 Vienna, Austria \\ Imahmoodian@compuserve.com, ${ }^{2}$ haring@ani.univie.ac.at
}

\begin{abstract}
We introduce an extended RSVP protocol called Mobile RSVP, which supports multimedia and real-time traffic in a Mobile IP based environment by providing certain levels of QoS guarantees. Our proposed protocol is appropriate for those mobile nodes that can not a-priori determine their mobility behaviour. We describe the conceptual design of the reservation mechanism of Mobile RSVP and discuss its overhead and control traffic. We further propose a dynamic resource partitioning policy and analyse the performance of Mobile RSVP in a single link based upon simulation results.
\end{abstract}

\section{Introduction and Related Works}

Current mobile and cellular networks and protocols only commit to limited QoS requirements. Consequently, they are not suitable for real-time and multimedia communications, which often require throughput and delay guarantees from the transport system. Nor are these protocols capable of adapting to variable and heterogeneous network conditions.

Recent research and development activities have been focusing on QoS issues in the wireline Internet. Protocols such as RSVP ([5,7]) provide simplex QoS guarantees based on the requirements of heterogeneous receivers by making hop-by-hop resource reservation along the communication path.

Talukdar and Badrinath try to consider the mobility aspect and propose in [4] a resource reservation protocol to support real-time applications of mobile hosts in an Integrated Services Packet Network. However, they assume that the mobility of a user is predictable so that mobility can be characterised precisely by a mobility specification consisting of a set of cells the mobile node is expected to visit during the lifetime of the communication session. Kam Lee has considered this shortcoming in [2] and proposes an anticipatory handoff control strategy relying on advanced establishment of so called branch connections to the neighbourhood of the mobile node. These branch connections are set up ahead of time in the surrounding of a mobile connection to support the hand-over process. However, no resources are reserved for or allocated to a branch connection when it is initialised. Resources are allocated after the completion of the hand-over process. We consider this fact to be the major drawback of the proposed framework since it is possible that required resources could not be provided when a mobile host visits a new location. In this case, the connection will be terminated.

Recognising the need of a protocol, which is able to provide QoS guarantees to mobile connections with heterogeneous requirements, we took a theoretical approach towards resource reservation in a mobile cellular network and propose the concept of an extended RSVP protocol, called Mobile RSVP, which is capable of providing QoS guarantees to mobile connections in a Mobile IP-based environment.

When a mobile node $(\mathrm{MN})$ initiates a session with a certain QoS guarantee by reserving link bandwidth along the path from the sender to its current location, the QoS guarantee is valid only in that location; when the MN moves to a new location, the QoS guarantee is not valid in the new location.

In this case a suitable protocol is required to maintain the reserved portion of the connection until a new connection is set-up, which is capable of providing QoS guarantees. The new protocol should also perform progressive resource reservation in advance, while the mobile host is travelling from cell to cell. 
This paper is organised as follows. In Section 2 we discuss general service models and introduce our Mobile RSVP protocol. In Section 3 we outline the protocol operation of Mobile RSVP and analyse various parameters. Section 4 presents our proposed dynamic resource sharing, which is also use in our simulation discussed in Sections 5 and 6. Section 7 presents various methods to enhance the performance of our proposed protocol. Section 8 discusses some open issues. We conclude in Section 9 with some future directions of this work.

\section{Mobile RSVP (A Proposal)}

In our proposed reservation protocol, in particular, we have considered non-deterministic mobility behaviour. Hence, our proposed protocol will enable a higher degree of flexibility for those mobile nodes, which might not be able to adhere to a pre-defined mobility specification.

Based on the above specification, we have proposed the Mobile RSVP protocol, which is an extension of RSVP standardised by IETF for reserving resources between Internet nodes. Mobile RSVP works with Mobile IP; consequently, the assumption is made that Mobile IP is known throughout the network.

We assume that route optimisation is employed in the underlying Mobile IP protocol to facilitate the correspondent node with an up-to-date mobility binding. It is further assumed that each cell is connected to the fixed network through a base station. In the proposed network architecture, base stations are considered as part of Mobile IP routers with the facility to communicate with and keep track of mobile nodes. Preferably, there should be a wired direct connection between neighbouring base stations. The bandwidth shall be divided into two portions: one for time constrained traffic on a reservation basis; and the other for connectionless non-timeconstrained traffic on a contention basis, basically for control information. Each base station co-ordinates and administers channel access and bandwidth reservation in a cell.

Mobile RSVP extends the functionality of PATH and RESV message to also perform passive reservation of resources and introduces two major new messages ACTIVATE and UPDATE to activate passive reservations and to update previous reservations in case of service degradation.

\section{Protocol Operation}

We assume that a mobility binding already exists to create an association between a home agent and the corresponding care-of address, along with the remaining lifetime of the association. Thus, datagrams can be tunnelled to the foreign agent and subsequently forwarded to the mobile node.

For simplicity, we consider a simplex unicast communication link between sender and receiver.

\subsection{Mobile RSVP Connection Set-up}

To avoid reservation of resources in the triangle route, a four-way handshake is proposed (Figure 1):

1. A traffic sender who aims to initiate a Mobile RSVP session sends a CONreq message. CONreq does not install reverse routing state in each router along the path, nor does it to provide receivers with information about the characteristics of the sender traffic and endto-end path. At this point, the home agent may deduce that the original source of the datagram has no binding update entry for the destination mobile node. According to the route optimisation specification, the home agent should send a binding update message to the original source node (correspondent node), informing it of the mobile node's current point of attachment. For a binding update to be authenticated by the original source node, we assume the corresponding node and the home agent to have established a mobility security association.

2. A traffic receiver willing to obtain (multimedia) data from the traffic sender responds to CONreq by a CONconf message. A CONrej is generated in case the receiver is not able or willing to be involved in a communication session with the traffic sender.

3. The sender sends a PATH message directly to the mobile node's care-of address, which travels hop-by-hop (downstream) through 


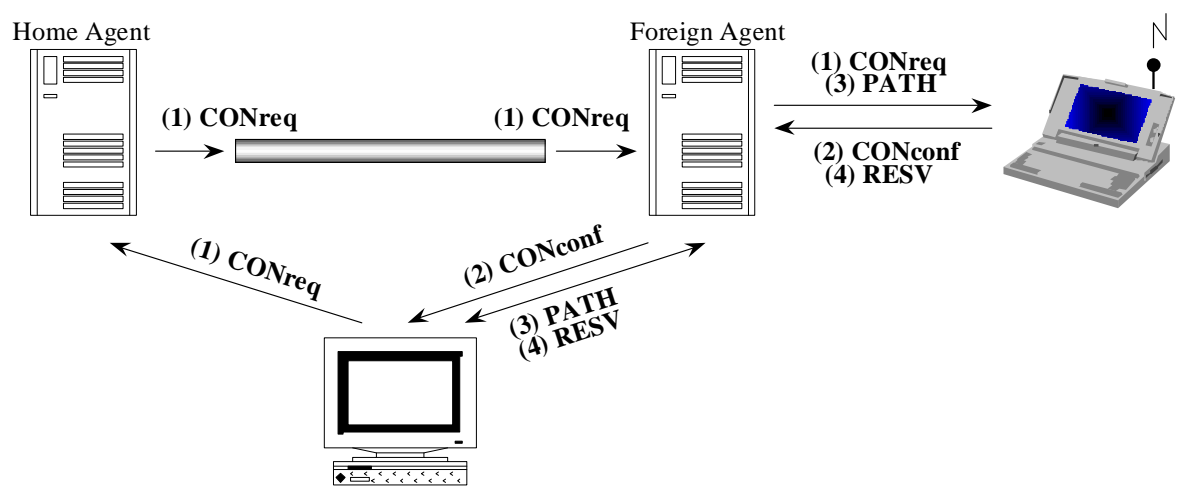

Figure 1: Mobile RSVP connection set-up.

subsequent RSVP routers installing (passive) QoS state in each router along the path.

4. Mobile node analyses the obtained PATH messages and generates a RESV message accordingly, which passes the routers in the reverse order (upstream). Each RSVP router has to evaluate the RESV message and make appropriate reservation.

\subsection{Progressive Resource Reservation}

Since the mobile node is roaming, each foreign agent has to make sure that resources are available as the relevant mobile node moves to the neighbouring networks. Therefore, a modified reservation model has to be performed, which is referred to as Progressive Resource Reservation. Based on the latter reservation model, a foreign agent acts as a traffic source and distributes PATH messages to the neighbouring base stations. PATH messages advise the neighbouring base stations of the characteristics of the sender traffic and install path state in corresponding nodes.

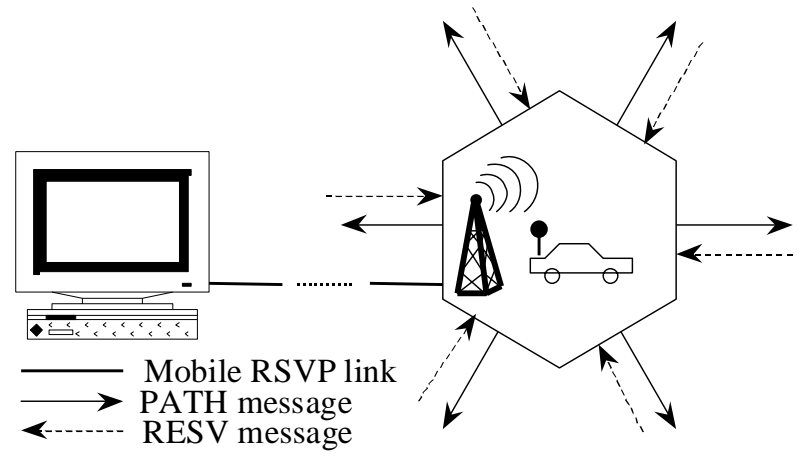

Figure 2: Progressive Resource Registration
Intermediate routers and corresponding base stations have to analyse the reservation request and either confirm or reject the request. In case of a confirmation, RESV messages are sent to the current foreign agent from each individual neighbouring base station (Figure 2). Every RESV message makes appropriate passive reservation along its corresponding branch.

The node that has initiated the reservation is assigned with the highest priority for his required resources. On one hand, this prioritisation ensures the mobile node that its reserved resources are available as soon as it enters the corresponding cell. On the other hand, it enables other mobile nodes to consume reserved but still unused (passive) resources. It is obvious that registered resources have to become available as soon as the mobile node with the highest priority requests them. Thus, the resource allocation is pre-emptive. Each (passive) reservation is associated with a certain lifetime $t_{\text {life }}$, after which it will be deleted. A foreign agent, who wishes to maintain the registration of its reservation request, has to refresh $t_{\text {life }}$, periodically. The introduction of $t_{\text {life }}$ will prevent the blocking of other requests being registered.

The potential reservation state of each individual path to the neighbouring base station along with the corresponding branch identification is maintained by the foreign agent. This information will be of use during hand-over.

\subsection{Hand-Over Procedure in Mobile RSVP}

The hand-over procedure of Mobile RSVP is based on the Mobile IP protocol. It assumes that 
a mobile node is successfully handed over to the adjacent base station and that the home agent has been notified accordingly.

\subsubsection{Activating Passive Reservation in the New Environment}

After the mobile node has changed its point of attachment, the previous foreign agent has also to hand-over QoS requirements to the new foreign agent.

The route optimisation draft of the Mobile IP protocol suggests that the former foreign agent has to keep record of the new care-of address of the mobile node for those packets that have been sent to the old care-of address while the mobile node was changing its point of attachment. Foreign agent keeps this information until an appropriate link is established between the correspondent node and the mobile node. Mobile RSVP also suggests activating adequate resources between two neighbouring base stations:

To change the state of a reservation from passive to active, the former foreign agent $F A_{l}$ in the cell $C_{l}$ (Figure 3 ) has to send an ACTIVATE message to the corresponding agent in the neighbouring cell $C_{2}\left(F A_{2}\right)$ along the same path, which has been established during the passive reservation. The correct path is identified through the relevant session identification. In case of a service degradation, which is determined by comparing the reservation state of the selected path (path to $F A_{2}$ ) with the current service specification, $F A_{l}$ has to inform all previous nodes involved in the session (including the traffic sender) and update their reservation states accordingly. This action is performed using an UPDATE message sent in the reverse path from the current foreign agent to the sender.

\subsubsection{Release and Record Resource Reservations}

When a mobile node moves to a new point of attachment, passive reservations in the old neighbourhood become obsolete. Hence, either they time out or they have to be released. For this reason, the old foreign agent distributes a RELEASE message to its neighbourhood (Figure 4a) informing them that the relevant registration is not valid any longer.

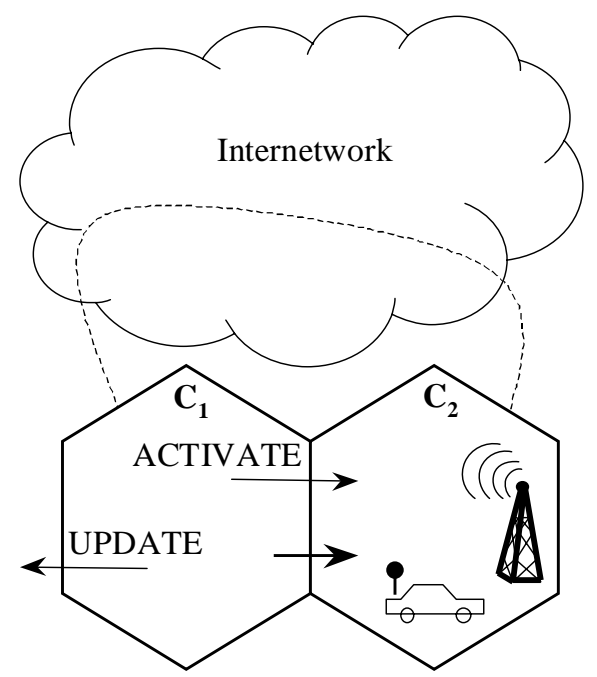

Figure 3: Hand-over procedure in Mobile RSVP

On the other hand, the new foreign agent is responsible to pre-advice (PATH message) its neighbourhood with regard to the arriving mobile node (Figure $4 \mathrm{~b}$ ). This will again initialise the progressive resource reservation and generate reservation requests.

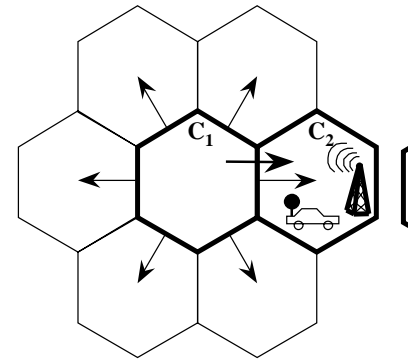

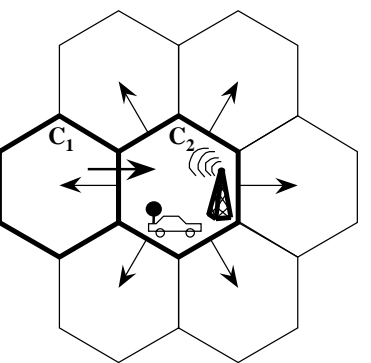

b)
Figure 4: Post hand-over procedure of Mobile RSVP

\subsubsection{New Mobile RSVP Link to the Current Care-of Address}

When a correspondent node capable of providing Mobile RSVP services receives an updated mobility binding, it may initiate a new Mobile RSVP link to mobile node's new point of attachment by sending a PATH message to the mobile node (Figure 5). However, establishing a 
new RSVP link is a matter of trade-off and has to be decided by the RSVP initiating entity.

At the same time, multimedia datagrams directed towards the mobile node are forwarded through the old foreign agent $F A_{l}$ to the new destination $F A_{2}$ of the mobile node along the Mobile RSVP connection between $F A_{1}$ and $F A_{2}$. Upon receiving the PATH message, $F A_{2}$ initiates a reservation by sending a correspondent RESV message. When the correspondent node receives the RESV message it automatically updates the mobility binding and starts sending datagrams to the $F A_{2}$, probably at a modified service quality. This also shows the flexibility of Mobile RSVP to adjust to variable network conditions.

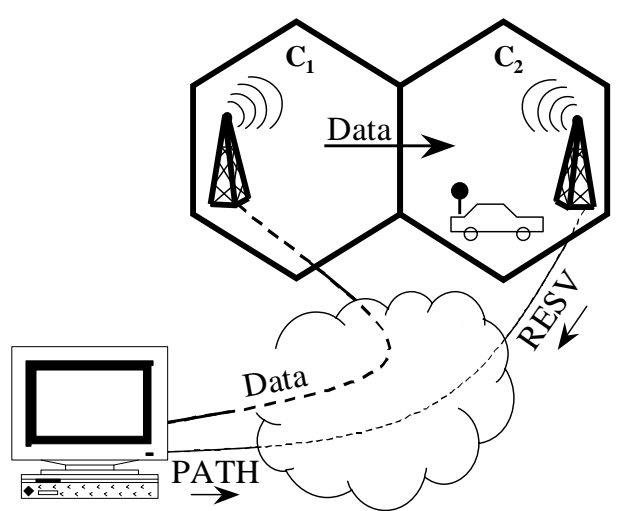

Figure 5: Creating a new Mobile RSVP link

The so-called "post-hand-over" procedure is realised in the Mobile RSVP layer of the corresponding protocol stack.

It is the responsibility of the Mobile RSVP instance to temporarily store updated mobility bindings until a new communication link with guaranteed QoS becomes available. Upon termination of a session, which is initiated by an END message, corresponding registrations are released based on the instruction of the relevant foreign agent.

\subsection{Protocol Analysis}

In this section, we try to calculate the response time of call set-up, connection hand-over and resource registration for a unicast mobile connection between a mobile node and a correspondent node. We also try to assess the amount of control traffic generated by Mobile RSVP.

The following parameters are used:
$H_{a} \quad$ Avg. number of hops along an active Mobile RSVP connection

$H_{c h} \quad$ Avg. number of hops between home agent and correspondent node

$H_{h f} \quad$ Avg. number of hops between home agent and foreign agent

$H_{\text {cell }} \quad$ Avg. number of hops between two foreign agents in neighbouring cells

$N_{\text {neighbour }} \quad$ Number of neighbouring cells

$\tau_{w} \quad$ Avg. transmission delay over a wired link

$\tau_{w l} \quad$ Avg. transmission delay over a wireless link

$\tau_{r} \quad$ Avg. routing delay in a router or base station

$\tau_{r d} \quad$ Avg. reservation processing delay in a router or base station

$\tau_{\text {caps }} \quad$ Avg. encapsulation or decapsulation delay of datagrams

\section{Response Time during Mobile RSVP connection set-up}

Let $t_{s}$ be the time it takes to establish a mobile connection, measured from the moment the mobile host makes the request until its acceptance. It is given by

$$
t_{s}=t_{1}+4 \tau_{w l}+H_{a}\left(3 \tau_{w}+2 \tau_{r}+\tau_{r d}\right),
$$

whereby $t_{1}=2 \tau_{\text {caps }}+\left(\tau_{w}+\tau_{r}\right)+\left(H_{c h}+H_{h f}\right)$, which includes the time for encapsulation and decapsulation of the CONreq message at both ends of the tunnel and the time to transmit and route the initial message from the correspondent node to the mobile node's current point of attachment.

\section{Response Time during Progressive Resource Registration}

Let $t_{p}$ be the time it takes to process a Progressive Resource Registration measured from the moment the current foreign agent initiates the event until its eventual establishment. It is given by

$$
t_{p}=N_{\text {neighbour }} H_{\text {cell }}\left(2 \tau_{w}+\tau_{r}+\tau_{r d}\right) .
$$

\section{Response Time during Mobile RSVP hand- over}

Let $t_{h}$ express the hand-over latency. If the mobile node is transmitting or receiving when it crosses cell boundary, then $t_{h}$ is measured from the moment the mobile node greets the new 
foreign agent until it can resume transmission or the switchover occurs at the crossover node. It is given by

$$
t_{h}=\left(\tau_{w}+\tau_{r d}\right) H_{a}+\tau_{w} H_{c e l l}+t_{n},
$$

where $t_{n}$ refers to the time it takes to establish a new Mobile RSVP link to the mobile node's new point of attachment, to release registered resources and to perform appropriate Progressive Resource Registration given by

$$
t_{n}=t_{p}+H_{\text {cell }} \tau_{w}\left(N_{\text {neighbour }}-1\right)+t_{\text {new }},
$$

whereby $t_{\text {new }}=H_{a}\left(2 \tau_{w}+\tau_{r}+\tau_{r d}\right)+2 t_{w l}$ and refers to the time to establish a new Mobile RSVP link from the correspondent node to mobile node's new point of attachment.

\section{Protocol Overhead and Control Traffic}

Classified in Table 1 are the protocol messages (including acknowledgements) generated in the events of connection establishment, Mobile RSVP hand-over and connection termination. In general, the amount of control traffic is linearly proportional to the number of neighbouring cells

$N_{\text {neighbour }}$.

Our proposed protocol generates even less control traffic when the reservation is initiated by the mobile node, i.e. the mobile node sends a PATH message to the corespondent node, provided the correspondent node is connected to a fixed ("wired") network. The a-priori knowledge of the correspondent node's address makes initial set-up messages CONreq and CONconf superfluous, since the mobile node does not need to go through the home agent to reach a correspondent node. In this case, the total number of control messages will reduce by four.

\section{Dynamic Resource Sharing}

Mobile RSVP distinguishes three different kinds of calls with defined priorities. These different types include best-effort calls, guaranteed calls and active hand-over calls, whereby hand-over calls have the highest priority and best-effort calls the lowest.

A dynamic resource-sharing algorithm is proposed to make use of the priority defined for each type of call. By adjusting the partitioning policy according to the actual flow of prioritised calls, it potentially allows every type of call to use the total resource capacity.

Dynamic resource partitioning demands that at any particular point of time $t$, the total number of active channels does not exceed the total number of available channels. As an implication the relation

$$
C_{\text {total }} \geq C_{i}
$$

should hold, whereby $C_{\text {total }}(t)$ represents the total number of channels at a particular point of time. Accordingly, $C_{i}(t)$ refers to the resources already assigned to each call class $i$.

We considering the three distinct classes of calls: best-effort, guaranteed and hand-over. Hence, in he above equation $C_{b}(t), C_{g}(t)$ and $C_{h}(t)$ indicate the total number of admitted best-effort calls, guaranteed calls and active hand-over calls, respectively.

By definition, the total number of channels reserved for passive hand-over calls $C_{h}^{\prime}(t)$ can never exceed the total number of channels $C_{\text {total }}(t)$ less the total number of channels assigned to active calls with QoS guarantees (i.e. $C_{g}$ and $C_{h}$ ). This is determined by the relation

$$
C_{h}^{\prime} \leq C_{\text {total }}-\left(C_{g}+C_{h}\right) \text {. }
$$

The above relation does not consider the number of channels allocated to best-effort calls. This implies that in case all available channels are utilised, passive resources can still be allocated as long as a sufficient portion of active channels belong to best-effort calls.

The three different type of calls are admitted according to their priority and the available resource capacity as follows:

Best-effort calls are admitted if there are sufficient idle channels available to satisfy their minimum resource requirement $\tilde{C}_{b, \text { min }}(t)$ as indicated by

$$
\tilde{C}_{b, \min } \leq C_{\text {total }}-\left(C_{b}+C_{g}+C_{h}\right) .
$$

Upon availability, channels may be allocated to best-effort calls up to their maximum resource requirement $\tilde{C}_{b, \max }(t)$.

Guaranteed calls are admitted if

$$
\tilde{C}_{g} \leq C_{\text {total }}-\left(C_{b}+C_{g}+C_{h}+C_{h}^{\prime}\right),
$$

where $\tilde{C}_{g}(t)$ refers to the total channel requirement of guaranteed calls. Since hand-over calls can not be interrupted or degraded, the 


\begin{tabular}{|c|c|c|c|c|}
\hline & $\mathbf{M N} \leftrightarrow \mathbf{F A}$ & $\begin{array}{l}\text { FA } \leftrightarrow \text { fixed } \\
\text { network }\end{array}$ & $\mathbf{F A} \leftrightarrow \mathbf{F A}$ & $\begin{array}{l}\text { Total } \\
\text { Number }\end{array}$ \\
\hline Connection set-up & $\begin{array}{l}1 \times \text { CONreq } \\
1 \times \text { CONconf } \\
1 \times \text { PATH } \\
1 \times \text { RESV }\end{array}$ & $\begin{array}{l}1 \times \text { CONreq } \\
1 \times \text { CONconf } \\
1 \times \text { PATH } \\
1 \times \text { RESV }\end{array}$ & $N_{\text {neighbor }} \times(\mathrm{PATH}+\mathrm{RESV})$ & $2 N_{\text {neighbor }}+8$ \\
\hline $\begin{array}{l}\text { Mobile RSVP } \\
\text { hand-over }\end{array}$ & & $1 \times$ UPDATE & $\begin{array}{l}1 \times \text { ACTIVATE } \\
N_{\text {neighbor }} \times \text { RELEASE } \\
N_{\text {neighbor }} \times(\mathrm{PATH}+\mathrm{RESV})\end{array}$ & $3 N_{\text {neighbor }}+2$ \\
\hline Post hand-over & $\begin{array}{l}1 \times \text { PATH } \\
1 \times \text { RESV }\end{array}$ & $\begin{array}{l}1 \times \text { PATH } \\
1 \times \text { RESV }\end{array}$ & $N_{\text {neighbor }} \times(\mathrm{PATH}+\mathrm{RESV})$ & $2 N_{\text {neighbor }}+4$ \\
\hline $\begin{array}{l}\text { Connection } \\
\text { termination }\end{array}$ & $1 \times \mathrm{END}$ & $1 \times \mathrm{END}$ & $N_{\text {neighbor }} \times$ RELEASE & $N_{\text {neighbor }}+2$ \\
\hline
\end{tabular}

Table 1: Control messages in the Mobile RSVP

admission control unit has to consider passive hand-over calls waiting to be activated, each time a new guaranteed call arrives.

Hand-over calls are admitted if

$$
\tilde{C}_{h} \leq C_{\text {total }}-\left(C_{g}+C_{h}+C_{h}^{\prime}\right),
$$

where $\tilde{C}_{h}(t)$ refers to the passive channel requirement of hand-over calls. When passive hand-over channels become active, there are either enough idle channels to be allocated to the hand-over call or relevant amount of channels have to be taken away from active best-effort calls.

In order to further clarify our proposed dynamic resource partitioning, a hypothetical scenario is discussed in the next section.

\section{Simulation}

Figure 6 illustrates the simulation model of the proposed reservation procedure of Mobile RSVP. At the call arrival port different kinds of calls arrive with a certain statistical distribution.

The admission control module decides whether a new call can be accepted. Its judgement policy is based on the (active or passive) resource requirements of the new call and the overall resource capacity of the link.

The passive reservations register maintains a list of all calls that will eventually visit current location and require resources. Each entry in the register indicates the time, at which the call becomes active, the amount of resources required, and the potential duration of resource consumption.

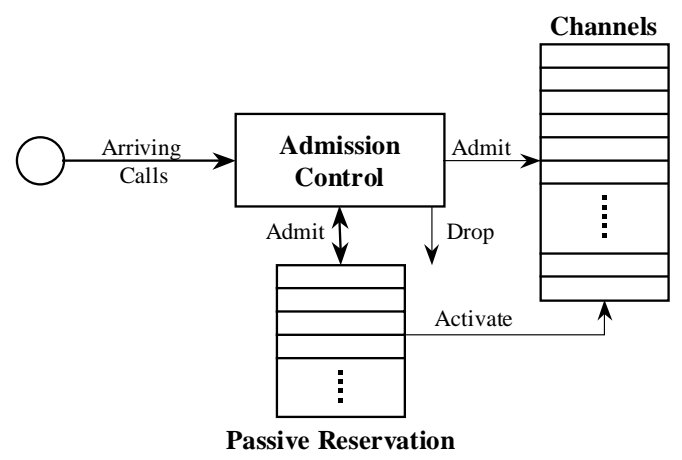

Figure 6: Simulation model of the reservation procedure of Mobile RSVP.

Finally, the total link capacity represented by a register of channels.

At the call arrival port three different kind of calls are distinguished:

Best-effort calls are admitted upon availability of bandwidth resources and do not enjoy any kind of QoS guarantees. These kinds of calls may even be blocked or terminated if the corresponding resources are required by those calls, which enjoy QoS guarantees.

Guaranteed calls initiate a new communication session for the first time at the current link. In contrast to best-effort calls, guaranteed calls enjoy QoS guaranteed in terms of a fixed bandwidth allocation for the whole lifetime of the connection at a the current link.

Unlike guaranteed calls, pre-advice or hand-over calls do not initiate a new communication session. They rather request resources to continue 
a session that was launched in one of the cells along the mobility path of the mobile node (usually the first cell). Hand-over calls are initiated by a cell, in which the mobile host currently resides. It is supposed to advise the relevant cell that it may be visited by the mobile node.

Once a guaranteed call is admitted, it has to be made sure not to disrupt the corresponding session until it is terminated. Therefore, special attention has to be paid to advance resource reservation requests, which aim to ensure the continuity of the connection during the session.

The total link bandwidth is divided into discrete units of channels. For each simulation run, a total link capacity is specified, which is represented by the total number of channels to be assigned to incoming calls. The total number of channels varies between 100 and 300 with an incremental step of ten.

Arriving calls have heterogeneous bandwidth requirements. In particular, the minimum bandwidth requirement $b_{\min }$ is uniformly distributed between 1 and 3 channels. Accordingly, the maximum bandwidth requirement $b_{\max }$ is distributed between 6 and 14 channels.

The effective channel holding time is exponentially distributed with a corresponding mean, which is defined in the start-up session of each individual simulation run and varies between 50 and 250 seconds with an incremental step of ten.

The time it takes for a passive hand-over call to become active (time to activate) is exponentially distributed with a corresponding mean, which is defined in the start-up session of each individual simulation run and varies between 50 and 250 seconds with an incremental step of ten. This definition is based on the assumption that any passive hand-over call will become active as soon as it leaves the previous cell, where it occupied resources with an effective channel holding time as specified above.

New calls arrive in Poisson manner independent of their type. The corresponding mean is in the range between 500 and 2500 calls per hour. The average no of calls is incremented by a factor of 100 during a simulation run. It is further assumed that two thirds of the arriving calls are best-effort calls. The remaining third is equally distributed between guaranteed calls and hand-over calls.
In our simulation, best-effort calls may be interrupted or degraded to the specified minimum number of channels, while guaranteed calls or hand-over calls can neither be interrupted nor degraded. Consequently, $b_{\min }$ and $b_{\max }$ are set the same for calls with QoS guarantees.

Figure 6 illustrates relevant activities related to reservation requests at four consecutive points of time.

Reservation requests are symbolised by $f(x, y[z])$, where $f$ refers to the type of call, $x$ and $y$ represent the (maximum) number of requested channels and the channel holding time, respectively. In case of (passive) hand-over calls, the optional $z$ indicates the remaining time to activate the call, which is determined using exponential distribution with the same conditions as channel holding time. We further assume the minimum bandwidth requirement to be one channel.

The channel register maintains an indexed list of active calls, where each channel is represented by one cell. The number in each cell refers to the remaining holding time.

At time $t_{l}$, a new best-effort call $b(2,3)$ arrives at the port requesting two channels with each three units of time to hold the channel. The two handover calls indicate a total reservation of five channels that are required upon activation. Only two channels are inactive and no channel holding time has expired yet. However, $b(2,3)$ can be admitted since there would be enough channels reserved for best-effort calls to cover an eventual activation of the hand-over calls.

At $t_{2}$, both the time to activate and the channel holding time of active channels are decreased by one time unit. A pre-advice call becomes active. In order to cover the required two channels, the best-effort calls $b_{3}$ and $b_{4}$ have to be degraded by one channel each.

At $t_{3}$, the hand-over call $h_{1}$ terminates. Furthermore, $b_{3}$ has to be degraded by one additional channel upon activation of the passive reservation $h(3,2)$ becomes active. Simultaneously, a new hand-over call $h_{p}(2,3,2)$ arrives, which can be admitted since sufficient best-effort calls exist to ensure resource availability.

At $t_{4}, b_{3}$ and $b_{4}$ terminate and two new calls $g(4,3)$ and $b(4,2)$ arrive at the port. According 

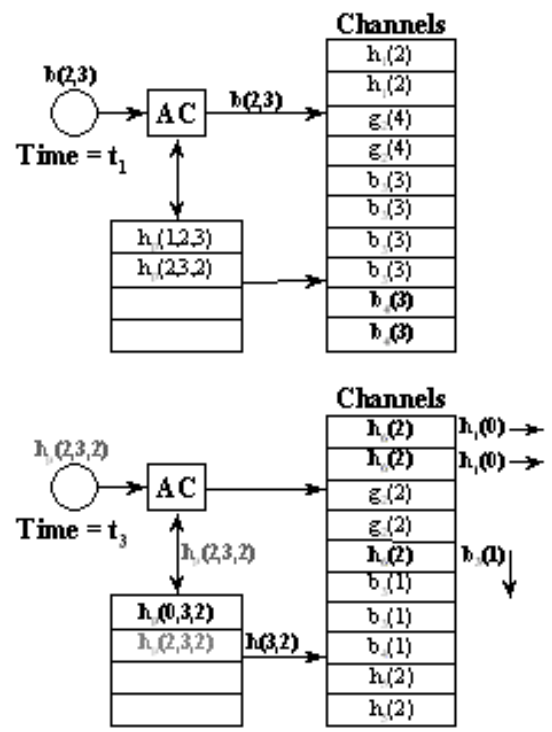

Figure 7: The operation of the simulation model.
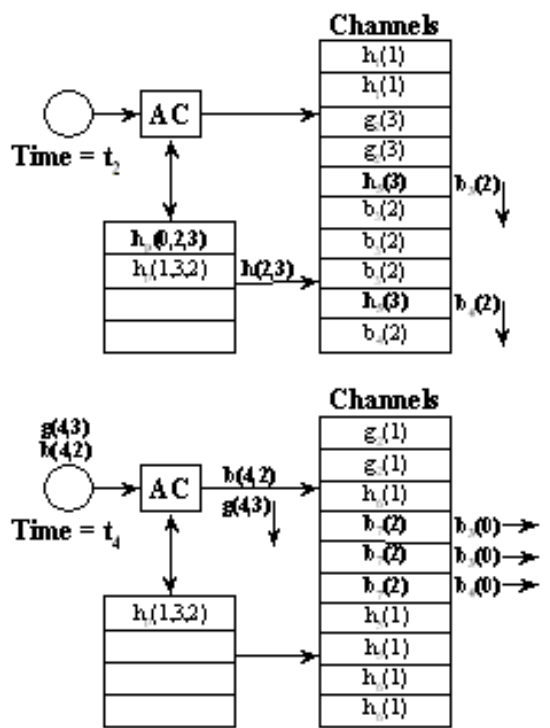

to the priorities given to the type of calls, $g(4,3)$, which symbolises a guaranteed call, is dealt first. However, it can not be admitted since there are not enough resources to satisfy the required four channels. The best-effort call $b(4,2)$ can be admitted since its defined minimum resource requirement of one channel is below the number of available channels at $t_{4}$.

\section{Simulation Results}

Simulations were run to analyse the effect of various parameters as described above on the behaviour of calls in a single cell.

In general, conditions were examined, under which the probability of blocked hand-over (preadvice) calls can be minimised.

In particular, we focused our research on the following probabilities:

- Blocked best-effort (BB) calls

- Blocked guaranteed (BG) calls

- Degraded best-effort (DB) calls

- Interrupted best-effort (IB) calls

- Blocked hand-over (BH) calls

The graph in figure 8 illustrates the effect of an increasing average number of calls/h on the blocking and dropping probability of calls when we assume the total number of channels and the mean channel holding time $\lambda_{t}$ (in seconds) to be fixed at 200 and 180, respectively. Trendlines represent the overall call behaviour.

The graph shows that the probability of DB calls undergoes an almost logarithmic increase at the beginning up to $60 \%$ of the total calls at an average rate of $1300 \mathrm{calls} / \mathrm{h}$. From there, the number of DB calls starts to oscillate between $50 \%$ and $60 \%$.

The probability of BB calls, however, increases almost linearly proportional to the average rate of calls/h.

The number of BG calls tends to a constant level of $16 \%$ of the total calls.

The trendlines of both $\mathrm{IB}$ and $\mathrm{BH}$ calls remain below $7 \%$ and $8 \%$, respectively, throughout the simulation.

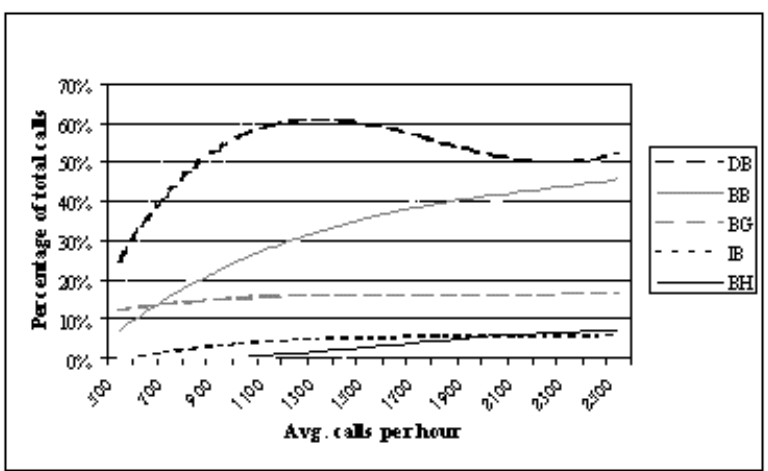

Figure 8: Call processing based upon avg. no. of calls

Figure 9 shows the effect of an increasing average channel holding time on the blocking and dropping probability of calls assuming the total 
number of channels and the mean number of calls/h to be constant $\lambda_{\text {calls }}$ at 200 and 1000 , respectively.

The mean channel holding time $\lambda_{t}$ is within the range between 50 seconds and 250 seconds.

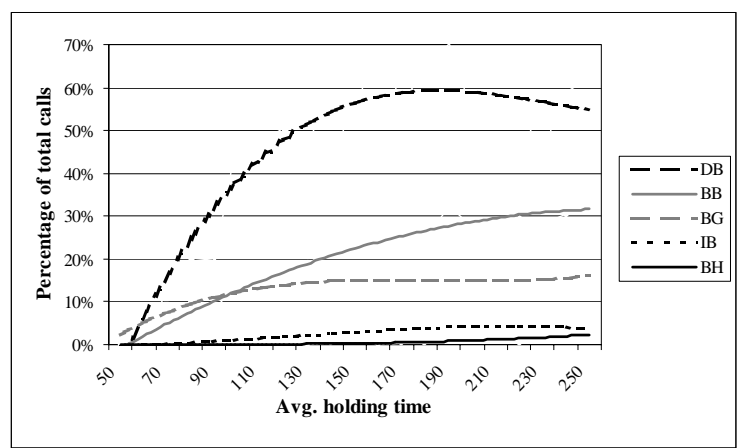

Figure 9: Call processing based upon avg. holding time.

The graph shows a growth trend for the probability of DB calls until $\lambda_{t}=190$. Above that level, the probability tends to fall.

The probability of BB calls, however, increases almost linearly proportional to the average channel holding time.

The polynomial trend of $\mathrm{BG}$ calls is almost tangent to the $15 \%$ level of the total calls.

The trendlines of both $\mathrm{IB}$ and $\mathrm{BH}$ calls remain below $4 \%$ and $3 \%$, respectively. The probability of BH calls can even be neglected if the average channel holding time is below 130 seconds.

In Figure 10, the effect of an increasing number of channels is illustrated. It is assumed that $\lambda_{t}$ and $\lambda_{\text {calls }}$ are 180 seconds and 1500, respectively. The number of channels varies between 100 and 300 .

The probability of DB calls examines a growth trend at the beginning and starts to be tangent to the $58 \%$ of total calls level just at the resource capacity of 220 channels.

The probability of $\mathrm{BB}$ calls declines almost indirect linearly proportional to the No of channels.

BG calls have an almost constant behaviour at about $18 \%$ of the total calls. Likewise,

IB calls show a constant trend at $5 \%$ of the total calls.

$\mathrm{BH}$ calls decline from $9 \%$ of total calls at 100 channels resource capacity to almost zero at 300 channels.

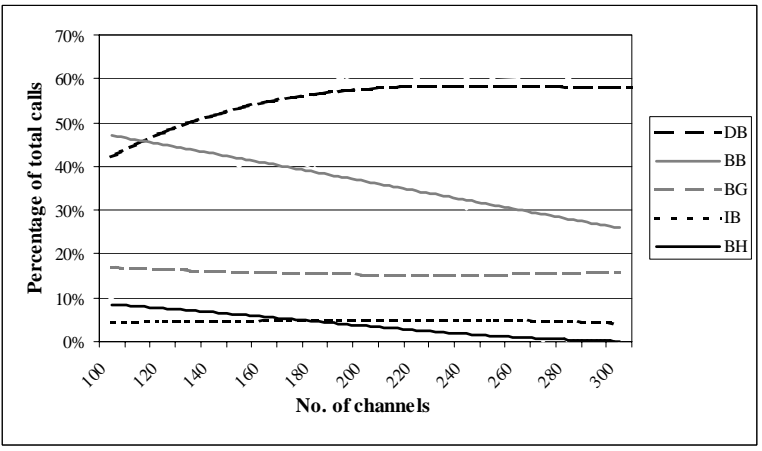

Figure 10: Call processing based upon no. of channels

\section{Performance Optimisation of Mobile RSVP}

As we have seen, the amount of control traffic increases linearly proportional to the number of neighbouring cells $N_{\text {neighbour }}$. Hence, one major source of improvement is to reduce the number or neighbours involved in a to communication session. One possibility is to take the mobility behaviour and the trajectories of mobile node into account. For example, it is quite unlikely that a mobile node return to the cell that it just left. Due to the capability of the model proposed in [10] to describe the user behaviour in detail, it can be applied to characterise the traffic in an individual single cell of a mobile network. Based on the proposed model, the probability of hand-over events and possible calls being blocked can be calculated. Using this model, it is also possible to estimate the distribution of channel holding times, which affects the number of refresh messages and the admission procedure.

Another possibility is to use the moving direction $(M D)$ strategy discussed in [11] for onedimensional micro-cellular systems. In these systems, forced call termination and channel changing occur frequently because of their small cell size. The MD strategy uses information on moving directions of the mobile units to decrease both the forced call termination blocking probability and the channel changing. An available channel is selected among those assigned to mobile units that are elsewhere in the service area and moving in the same direction as the concerned mobile users. The search for such a channel starts from the nearest non-interfering 


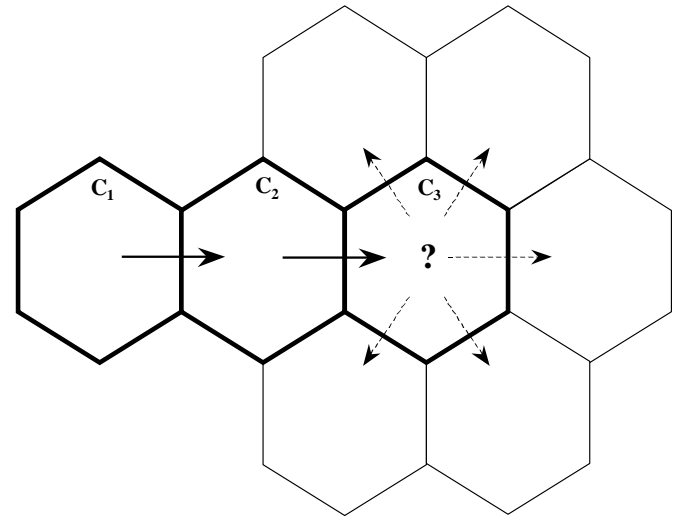

a)

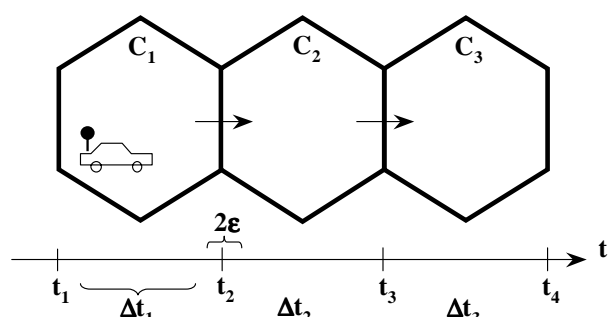

b)

Figure 11: a) Assessing the mobility behaviour of the mobile node based on the previous motion path. b) Resource reservation model for mobile nodes with deterministic behaviour.

cell to the one where the new call was initiated and stops at the cell that is a reuse distances away, where $a$ is a parameter.

A foreign agent can also determine the direction of motion of mobile nodes from the sequence PATH messages associated with it (Figure 11a).

This information can be used by the foreign agent to decide whether to proceed Progressive Reservation Registrations, or it can be used by the network to adapt algorithms such as the Adaptive Prioritised Link Partitioning proposed by [2]. The result would be less control overhead and better network efficiency.

Another approach would be (and this applies to the policy control module of RSVP) the implementation of a priority mechanism that allows users to send reservation requests with higher priority than others. The Integrated Services (IS) may be coupled with a billing system that charges the user according to priority level of his reservation request.

An a-priori knowledge of the mobile node's mobility behaviour, where the corresponding foreign agents are pre-determined might help to reduce control traffic and improve service availability. In fact, for this kind of applications Mobile RSVP does not represent an appropriate

\footnotetext{
${ }^{1}$ By taking advantage of physical characteristics of the radio environment, the same channel can be reused simultaneously by many sets of wireless terminals if they are spaced sufficiently apart. All such sets, which use the same channel, are referred to as co-channel sets or simply co-channels. The minimum distance at which co-channels can be reused with acceptable interference is called the co-channel reuse distance.
}

reservation model. In such cases, it is more convenient to perform resource reservation in a timely manner, where QoS can be guaranteed starting at a particular point of time and lasting for a certain period.

Hence, reservation has to be made in advance when the mobile node decides to leave its home network, so each foreign agent knows approximately when a mobile node will arrive in the respective cell (network) and how long it plans to stay. Foreign agents might be notified of any potential delay that might occur. This reservation model is best applicable if the mobile node is travelling for example by car or train, where the mobility route is somehow predetermined. However, it does not provide the flexibility a mobile node might require, but is exemplifies an equitable approach for a certain class of mobility behaviour.

Figure $11 \mathrm{~b}$ illustrates such a reservation model. In deterministic mobility behaviour model the equation

$$
t_{\text {total }, i}=\Delta t_{i}+2 \varepsilon
$$

should hold, where $t_{\text {total }, i}$ is total time a reservation should be maintained in the corresponding cell $i$ for a specified mobile node and $\Delta t_{i}=t_{i+1}-t_{i}$, which is the time the mobile node will occupy cell $i . \varepsilon$ refers to the average time required for hand-over. $2 \varepsilon$ is required to enable a smooth hand-over and depends upon the corresponding hand-over algorithm.

As already mentioned, IPv6 provides more sophisticated flow control and QoS provision mechanisms. Also, mobility support concepts 
have already been drafted and proposed in [1]. A liaison with Mobile IPv6 might improve the performance of Mobile RSVP. Nevertheless, this will remain a subject for further research Another important extension will be (this also applies to the policy control module of RSVP) to implement a priority mechanism that allows users to send reservation requests with higher priority than others. The integrated services may be coupled with a billing system that charges the user according to the priority of the relevant reservation request.

\section{Open Issues}

A major problem of Mobile RSVP will be its applicability in the current network infrastructure. It is required that relevant routers of the underlying network are aware of Mobile RSVP, otherwise the control traffic will be very high and will even affect the best-effort performance. As a first approach, it is suggested that Mobile RSVP be realised in corporate Intranets to provide multimedia or other real-time data to mobile nodes.

Another pitfall of Mobile RSVP is its control traffic and protocol overhead, which increases rapidly when both communication counterparts are mobile hosts. In case the correspondent node is mobile himself, a huge amount of additional control messages is necessary, which would make Mobile RSVP quite inefficient, since the underlying network structure might get overloaded with required protocol overhead.

In this case, both involved parties (mobile hosts) have to employ progressive resource registration. This will extremely increase the complexity of a potential mobile resource reservation protocol.

In a multicast scenario, the control traffic will be even higher. Appropriate measures have to be considered to overcome this pitfall.

\section{Concluding Remarks}

This work has addressed issues related to quality of service for multimedia applications in a nomadic Internet-based environment. A detailed analysis of the problems and requirements has yielded to recommendations, which have been adopted in the proposed reservation protocol and the relevant resource-sharing algorithm.
Our design is oriented towards mobile users, who (due to the characteristics of their multimedia applications) require certain quality of service guarantees, while they are travelling from location to location. In order to ensure the continuation of the required service in such an environment, resources have to be reserved in the immediate neighbourhood of mobile user's current location.

Our proposal complements other approaches by considering their weakness and disadvantages. Thus, it does not create unnecessary traffic, and performs appropriate advance resource reservations without an a-priori knowledge of the mobility path. This type of resource reservation is convenient for those mobile users requiring utmost flexibility.

In addition, the proposed resource-sharing algorithm further supports the continuity of service guarantees to mobile users.

The performance of our protocol in conjunction with the proposed resource-sharing algorithm has been analysed by simulating the overall behaviour in a single link. Simulation results show the continuity of service guarantees to admitted mobile users can be ensured at the expense of new calls and those calls receiving best-effort service.

Our further research will focus on the integration of user mobility aspects in our protocol. By predicting the mobility path we are able to reduce the number of passive reservations and control traffic.

Another issue that needs to be tackled is multicasting. As part of our future work, we will extend Mobile RSVP with the multicasting feature.

As already mentioned, we have also initiated research activities to evaluate a liaison between Mobile IPv6 [1] and RSVP, since IPv6 provides more sophisticated flow control and QoS provision mechanisms. We also plan to investigate and research the provision of mobility capabilities in Differentiated Services [9] networks.

\section{References}

[1] David B. Johnson and Charles E. Perkins, "Mobility Support in IPv6", Internet Draft, 
draft-ietf-mobileip-ipv6-08.txt, June 25, 1999.

[2] Kam Lee, "Supporting mobile multimedia in integrated services networks", Wireless Networks, Vol. 2, pp. 205-217, 1996.

[3] Charles E. Perkins, "Mobile IP, Adding Mobility to the Internet", MobiCom'97, The Third Annual ACM/IEEE International Conference on Mobile Computing and Networking, September 26, 1997.

[4] Anup K. Talukdar, B. R. Badrinath and Arup Acharya, "On Accomodating Mobile Hosts in an Integrated Services Packet Network", IEEE Proceedings of the Infocom 97, April 1997, pp. 1064-1053.

[5] Paul P. White, "RSVP and Integrated Services in the Internet: A Tutorial", IEEE Communications Magazine, May 1997, pp. 100-106.

[6] Charles E. Perkins and David B. Johnson, "Route Optimization in Mobile IP", Internet Draft, available at draft-ietf-mobileipoptim-08.txt, February 25, 1999.

[7] R. Braden, L. Zhang, S. Berson, S. Herzog and S. Jamin, "Resource ReSerVation Protocol (RSVP), Version 1 Functional Specification", RFC 2205 , available at ftp://ds.internic.net/rfc/rfc2205.txt.

[8] C. Metz, "IP QoS: Travelling in First Class on the Internet", Internet Computing, Vol. 3, Nr. 2, pp. 84-88, March/April 1999.

[9] S. Blake et al., "An Architecture for Differentiated Services", RFC 2475, available $\mathrm{ftp} / / / \mathrm{ds}$.internic.net/rfc/rfc2475.txt.

[10] Plamen I. Bratanov, "User Mobility Modelling in Cellular Communications Networks", Dissertation Thesis, Vienna University of Technology, February 1999.

[11] I. Katzela and M. Naghshineh, "Channel Assignment Schemes for Cellular Mobile Telecommunication Systems: A Comprehensive Survey", IEEE Personal Communications, June 1996, pp. 11-31. 